\title{
CONSTRUCTION AND PERFORMANCE OF THE ELLIPTICAL POLARIZATION UNDULATOR EPU5.6 IN SRRC
}

\author{
C.H. Chang, H.H. Chen, T.C. Fan, Jenny Chen, C.S. Hwang ${ }^{+}$, M.H. Huang, K.T. Hsu, F.Y. Lin, C.D. Li, H.C. Liu, \\ Ch. Wang, SRRC, Hsinchu, TAIWAN
}

\section{Abstract}

A 132 pole elliptically polarizing undulator (EPU5.6) with magnetic period of $5.6 \mathrm{~cm}$ for production of brilliant and soft $\mathrm{x}$-rays has been constructed and performed the field measurement at Synchrotron Radiation Research Center (SRRC). The "Apple-II" type device was selected for this EPU5.6 structure. The key factors in the construction and assembly process stage will be described. In addition, the shimming algorithm for the magnetic field adjustment, the performance of the mechanical structure, magnetic field distribution without the field shimming will be presented in this paper. Meanwhile, the control performance also will be discussed.

\section{INTRODUCTION}

A 3.9-m long elliptical polarized undulator EPU5.6 has been constructed and field shimming was done for creating a high quality and high photon flux at SRRC. Two diagonal magnet arrays were fixed and the other diagonal arrays were free to be moved to create the right/left circular polarization. Therefore, this EPU5.6 [1] was only used for producing the linear polarized on vertical/horizontal and the right/left elliptical, circular polarization light. However, if the two fixed arrays are free, it can also create the linear polarization from $0^{\circ}$ to $180^{\circ}$ [2]. When it is operated under the $1.5 \mathrm{GeV}$ SRRC storage ring, it will radiate $80-1500 \mathrm{KeV}$ circularly and elliptically polarization light for the X-ray Magnetic Circular Dichroism (XMCD) experiments or other photon absorption experiment to analyze the magnetic material.

In light of above circumstances, the magnet sorting and shimming technique must be developed to maintain the same quality of field and spectrum in different phasing modes for various polarization lights. Consequently, the magnetic field strength of $B_{y}$ and $B_{x}$ can be obtained by the 3-D Hall probe measurement system [3] to correct the trajectory and optical phase as well as the roll-off of each pole. Meanwhile, the integral field strength of $\int B_{y} d s$ and $\int \mathrm{B}_{\mathrm{x}} \mathrm{ds}$ can be obtained by the long-loop-coil measurement system with the dynamic measurement method [4] to modify and maintain the harmonic field components within the specification.

${ }^{+}$Email: cshwang@ srrc.gov.tw

\section{MECHANICAL ASSEMBLY AND ACCURACY TESTING}

It is a 3.9-m long undulator and it is very difficult to get a high mechanical accuracy. Therefore it is very important to shim field for the field quality control. In addition, the magnet assembly is a key factor to maintain a good field quality. For maintaining a high quality mechanical system, two arrays of the diagonal pair were fixed and the other two arrays can be moved to change the phase.

\subsection{Magnet block assembly}

The magnet block should be very careful to be assembled on the keeper to avoid the mechanical error. Each magnet blocks of the magnetized direction in vertical/horizontal were glued on the individual holder by the epoxy. Meanwhile, the magnet block was fixed on the holder by the clamp [1]. Finally, the holder was fixed on the base plate by the stainless steel screw. This assembly process will be easy to create the mechanical errors that are the magnet tilt, horizontal or vertical position variation. These errors are mainly due to the strong clamping force, which is very difficult to be controlled. For solving this problem, an assembly fixture for gluing and clamping the block on the keeper is necessary for each block assembly. Each keeper can be adjusted in horizontal/vertical position on the base plate to tune the magnetic field.

\subsection{Support system and backing beam assembly}

The backing beam is made of stainless steel and the sliding beam is made of aluminum. Hence, when assemble the sliding beam on the backing beam that was fixed on the $\mathrm{C}$-frame, the different thermal expansion coefficient on the bimetal material will induce a large mechanical distortion, especial on the long undulator which is larger than $2 \mathrm{~m}$ long. Therefore, we will be careful to control the tunnel temperature within $0.5^{\circ} \mathrm{C}$.

\subsection{Sliding beam of the driving structure}

This part is also a crucial issue for the field quality control when change the phase. If the sliding beam structure on the backing beam is not sufficient rigid, especially the cross roller bearing between the upper or lower two arrays, to overcome the repulse or attractive force between the four arrays on the different phase. The harmonic integral field components and peak field will be 
changed significantly in different phase position. Meanwhile, the sliding beams are guided by linear bearings that travel on the guide rails. These assemblies of these guided beams between the sliding beam and backing beam will be very careful to be adjusted to maintain the (1) good straightness, (2) parallel between each guide rail, and (3) the phasing direction should be parallel to the backing beam surface plane.

\subsection{Mechanical performance}

Using the laser interferometer and dial gauge in different phase position and gap to measure the mechanical performance. The $1 \mathrm{~mm}$ gap variation (The gap variation due to force difference on different phase position) between the two arrays on the lower/upper beam can be maintained within $\pm 20 \mu \mathrm{m}$. The reproducibility of the phasing and gap moving can be controlled within $\pm 1 \mu \mathrm{m}$ by the feedback control system. When the magnet gap was operated in both direction, open and close loop, the backlash is about $10 \mu \mathrm{m}$. Temperature difference induce the mechanical distortion error (like as the sag distortion) of the backing beam will be $0.03 \mathrm{~mm} /{ }^{\circ} \mathrm{C}-\mathrm{m}$, it is a significant amount to induce the field error for a long undulator. The tilt error on the vertical direction that is due to the unparalleled between the magnetic field axis and the phasing axis is about $50 \mu \mathrm{m}$.

\section{FIELD SHIMMING ALGORITHM}

When the 1-m prototype EPU5.6 was completed, we have revised a little about the shimming algorithm. We apply the shimming code to correct the four arrays individually. And then combine the magnetic field distribution of each magnet arrays on the lower or upper beam by means of the field measurement data of each array to simulate the optical phase and trajectory on different phase. The simulation method is to combine the two or three, even four array magnetic field strength (Because the magnetic field of the pure magnet structure is suited to use the superposition theorem). For the simulation purpose, the field measured interval distance should be smaller than 1 $\mathrm{mm}$ and the position precision should be within few micrometers. When using this shimming code to correct the field strength deviation, the difference of average peak field and half period integral strength between the four arrays should be kept within $0.5 \%$ which is corresponding to the common on-axis field. These simulation results will give us which pole belongs to whish's array should be shimmed. Finally, the combination of the four magnet arrays to simulate the phase error and the trajectory distribution to fine-tune the field error. When fine-tune is completely, the four arrays were assembled on the sliding beam to measure the magnetic field distribution. Based on the field-measured results and the shimming algorithm to correct the uncorrected trajectory and phase error, which are induced from the mechanical and simulation error of the entire magnet. Finally, the end pole was used to tune the fringe field effect. The trim magnets, which are located at the both end sides, were used to adjust the integral multipole field of By and Bx. Four air coils located at both ends to correct the horizontal/vertical axis trajectory (in the up/down stream) and offset which are on the different polarization mode.

\section{MAGNETIC FIELD MEASUREMENT AND PERFORMANCE}

The features of the peak magnetic field distribution of $\mathrm{B}_{\mathrm{y}}$ and $\mathrm{B}_{\mathrm{x}}$ are measured at the circular polarization condition and revealed in Fig. 1, which was measured before the magnetic field shimming.

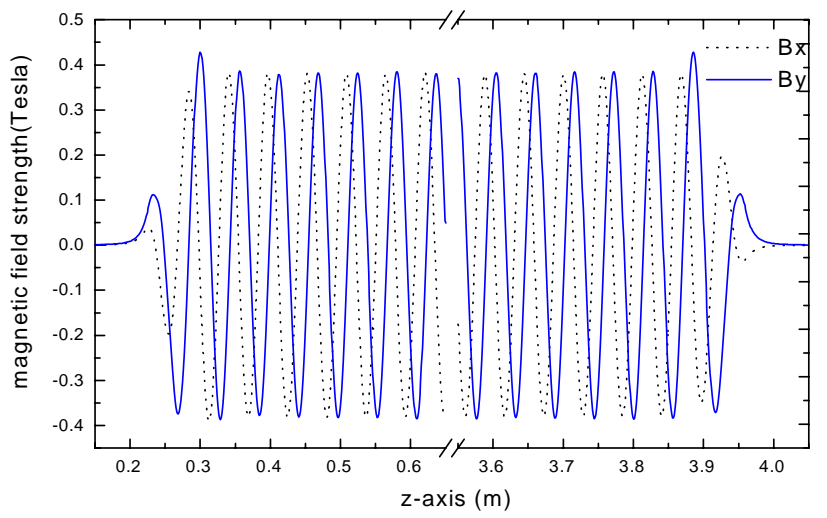

Figure 1: The magnetic field distribution of $B_{y}$ and $B_{x}$ along the longitudinal axis. Here exist a phase difference $\pi / 2$ between $B_{y}$ and $B_{x}$.

The scale of the roll-off range of the $\Delta \mathrm{B}_{y}(\mathrm{x}, \mathrm{y}) / \mathrm{B}_{\mathrm{y}}(0,0)$ and $\Delta \mathrm{B}_{\mathrm{x}}(\mathrm{x}, \mathrm{y}) / \mathrm{B}_{\mathrm{x}}(0,0)$ at the circular polarization condition are measured and shown in Fig. 2 and 3. These figures reveal that the roll-off range seems very short at the circular polarization condition, especially in the vertical axis. If the field strength deviation was kept within $\pm 0.5 \%$, the roll-off range in the vertical axis is about $\pm 1.2 \mathrm{~mm}$ of the vertical

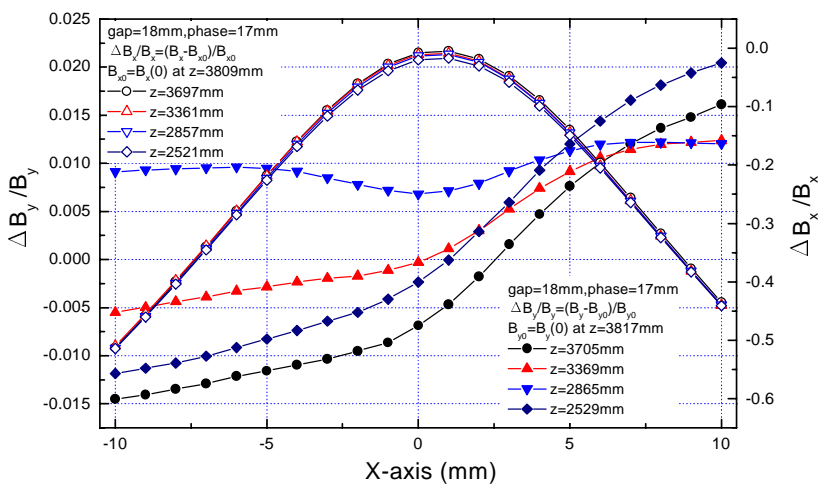

Figure 2: The roll-off range of the magnetic field distribution of $B_{y}$ and $B_{x}$ without shimming along the transverse $\mathrm{X}$-axis. 


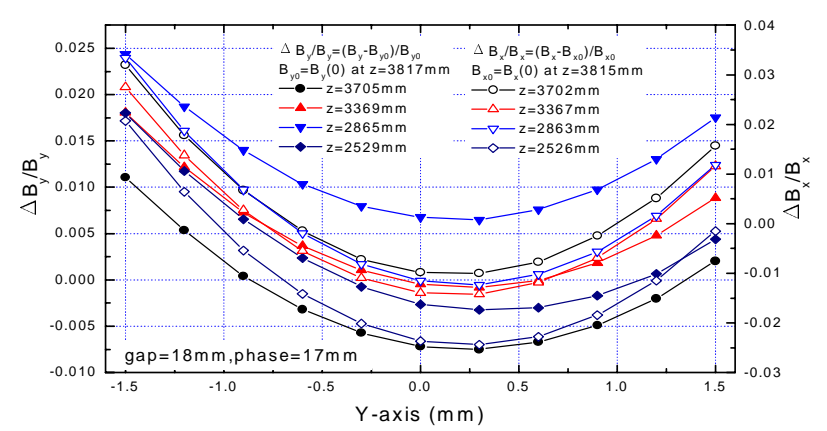

Figure 3: The roll-off range of the magnetic field distribution of $\mathrm{B}_{\mathrm{y}}$ and $\mathrm{B}_{\mathrm{x}}$ without shimming along the vertical y-axis.

field $\mathrm{B}_{\mathrm{y}}$ and $\pm 0.8 \mathrm{~mm}$ of the horizontal field $\mathrm{B}_{\mathrm{x}}$ (See in Fig. 3 ). If the field strength deviation was kept within $\pm 0.25 \%$, the roll-off range in the horizontal axis is about $\pm 10 \mathrm{~mm}$ of the vertical field $\mathrm{B}_{\mathrm{y}}$ and $\pm 1 \mathrm{~mm}$ of the horizontal field $B_{x}$ (See in Fig. 2). This roll-off range is much smaller than the convention undulator. Such a short roll-off range is the crucial issue of the "Apple II" structure, especially the roll-off range of horizontal field $B_{x}$. Due to the problem, the real magnet center should be aligned accurately to be in the electron closed orbital plane of the storage ring. Otherwise, the photon flux will decrease much more. Fig. 2 also shows that the roll-off region is big difference on each pole. Therefore, it is a big trick task to correct the roll-off of each pole on the entire magnet to be within $0.5 \%$, especially in the horizontal axis.

The integral field deviation on different phase and gap are also measured and revealed in Fig. 4 and 5. Measurement results show that the on-axis integral field strength was changed on different phase, but the variation of integral strength $B_{y}$ and $B_{x}$ can be maintained within $70 \mathrm{Gcm}$ (see in Fig.5). Meanwhile, integral field variation and the profile of $B_{x}$ in the central region (within $\pm 10 \mathrm{~mm}$ ) on transverse axis also maintain within $100 \mathrm{Gcm}$ in different gap (see in Fig.4). But the vertical integral field was changes much more than the horizontal field (see in Fig.4). This big variation can be controlled within 100 $\mathrm{Gcm}$ by the end pole and trim magnets adjustment.

\section{CONCLUSION}

Until now, the field features without any field shimming of this undulator seems not too bad. However, we have found several problems, (1) Mechanical stability is very difficult to be controlled in such a long backing beam, especial in a bimetal combination of the backing beam and sliding beam (This factor will produce the $0.3 \mathrm{~mm}$ mechanical distortion of each backing beam), (2) The horizontal phase moving plane is not parallel to the magnetic field axis (about $40 \mu \mathrm{m}$ ), (3) the peak field and integral strength variation of each pole and half period have a big difference between the different phase (The rms field strength deviation will increase from $0.3 \%$ to
$0.8 \%$ ). These problems should be solved in the near future.

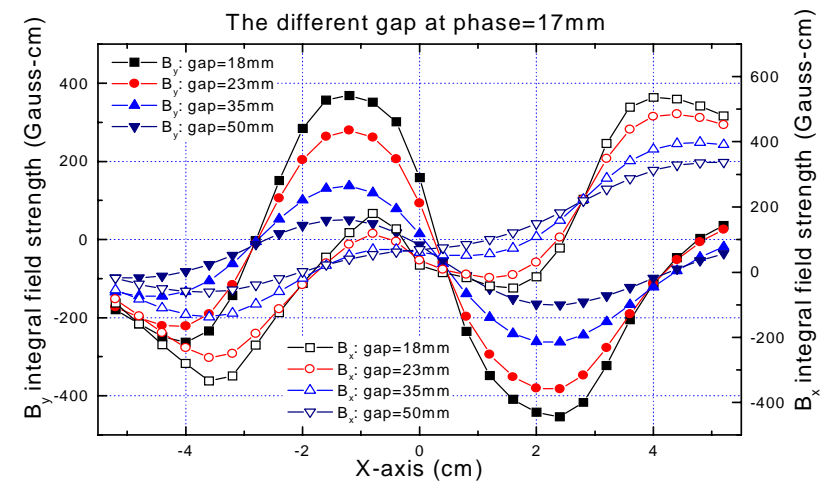

Figure 4: Integral field strength and profile on the transverse axis in different gap without shimming. Phase was fixed at the circular polarization condition.

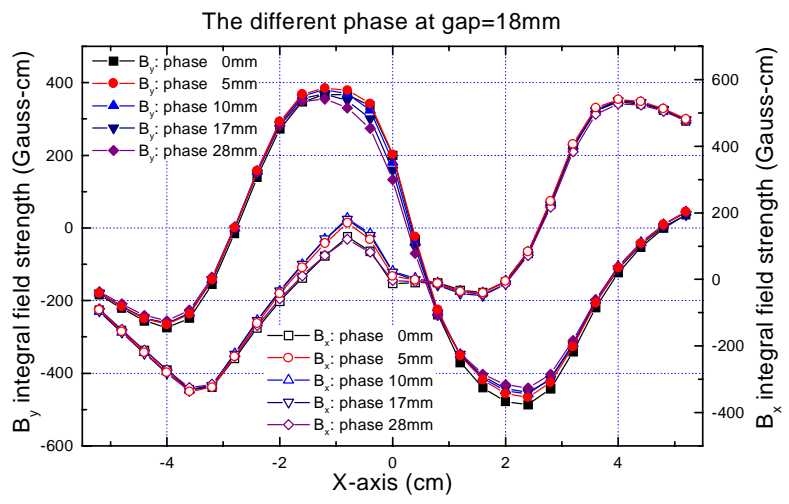

Figure 5: Integral field strength and profile on the transverse axis in different phasing position without shimming. Gap was fixed at the minimum gap of $18 \mathrm{~mm}$.

\section{ACKNOWLEDGMENTS}

The authors would like to thank the National Science Council of Taiwan under the contract No. NSC88-2112M-213-008. Director C.T. Chen (SRRC) and Dr. J.R. Chen (SRRC) are appreciated for supporting this project.

\section{REFERENCES}

[1] C.H. Chang, et al, "Optimization design for SRRC elliptically polarizing undulator", present in MT-15 (1997).

[2] C.S. Hwang, Shuting Yeh, "Various polarization features of a variably polarized undulator with different phasing modes", Nucl. Instr. and Meth. A420 (1999)29-38.

[3] C.S. Hwang, et al., "Advanced Field-Measurement Method with Three Orthogonal Hall Probes for an Elliptically-Polarizing Undulator", J. Synchrotron Rad. (1998). 5, 471-474.

[4] C.S. Hwang, et al., "The long loop coil measurement system for the insertion device", Asia Particle Accelerator Conference (APAC'98), Japan, 1998. 